\title{
Anorexia nervosa complicating inflammatory bowel disease
}

\author{
P Mallett, S Murch
}

\begin{abstract}
Two cases of inflammatory bowel disease, occurring in adolescence and complicated by anorexia nervosa, are presented. The management of the bowel disease with corticosteroids appeared to precipitate the eating disorder in one case whereas covert withdrawal of steroid treatment led to life threatening complications of inflammatory bowel disease in the other. The difficulties of managing two serious conditions, each ideally treated in a specialist centre, are discussed and the dangers of treating adolescents with shape modifying drugs are highlighted.
\end{abstract}

Inflammatory bowel disease is now increasingly recognised in children and adolescents, although it is still underdiagnosed. ${ }^{12}$ The incidence of anorexia nervosa is unknown, but in cases with prepubertal onset, the ratio of male to female sufferers is much higher than for older age groups and the diagnosis is often missed. ${ }^{3} \mathrm{We}$ present two cases of childhoood anorexia nervosa, one in a boy who subsequently developed ulcerative colitis and the other in a girl whose Crohn's disease was complicated by the eating disorder. In both these cases corticosteroid treatment played a pivotal part and the management of one condition was profoundly and adversely affected by the other.

\section{Case reports}

CASE 1

A 14 year old Iranian boy was admitted to his local hospital with a short history of abdominal pain and diarrhoea. Ulcerative colitis was diagnosed by sigmoidoscopy and biopsy and he was treated with oral prednisolone, sulphasalazine being contraindicated because of glucose 6phosphate dehydrogenase deficiency. He was referred for further assessment because of continued symptoms, the diagnosis being confirmed at colonoscopy where moderate, predominantly distal disease was seen with biopsy specimens showing classical ulcerative colitis. $\mathrm{He}$ was stabilised on a mixture of oral and rectal steroids.

At referral his height lay on the 90 th centile with weight just below the 50 th centile $(49 \mathrm{~kg}$ ). Examination showed him to be in early puberty. During follow up it became apparent that he was steadily losing weight and he was referred for psychiatric assessment. It transpired that he had been eating abnormally since the age of 12 after remarks made by girls at school about his weight. Two months before the onset of his abdominal symptoms he had apparently weighed
$63 \mathrm{~kg}$ on his home scales and had determined to lose weight. He competed with his sister in this, developing a regime of intensive daily exercise and keeping a diary of food intake. He became obsessed by food, feeling that he had lost control if he did eat. He indulged with his sister in days of binge eating, after which she would induce vomiting and he would punish himself by increasing his exercise regime. He had undoubted disturbance of body image, feeling that his shoulders were becoming larger and more manly in the face of an emaciated appearance and the loss of more than $30 \%$ of his ideal body weight.

During the following months he became resentful of his steroid treatment as he felt it disturbed the shape of his face (he particularly wanted his cheekbones to be prominent) and he secretly stopped it. His colitis relapsed quickly and he was readmitted as an emergency with severe abdominal pain and bloody diarrhoea. His haemoglobin concentration had dropped to $80 \mathrm{~g} / \mathrm{l}$ and colonoscopy now showed a severe pancolitis. Despite high dose intravenous steroids he deteriorated, developing toxic megacolon that required emergency subtotal colectomy with ileostomy. Postoperatively he was concerned about the energy in his dextrosesaline infusion. His weight had dropped to a nadir of $32 \mathrm{~kg}$, a $50 \%$ fall in 10 months.

His subsequent progress has been complicated by ileostomy dysfunction, secondary sodium depletion, and by several episodes of subacute obstruction. Attempts to admit him to two adolescent psychiatric units were refused because of the complexity of his medical problems. Planned transfer to a third unit was postponed because of recurrent abdominal pain. Attempts to establish a strict behavioural regime on the general paediatric ward were unsuccessful in increasing his weight as he was adept at feigning compliance while actually avoiding food and covertly exercising.

His subsequent psychiatric management has been conducted largely as an outpatient, both at this hospital and by referral to a department of adolescent psychiatry. He has attended with his parents and sister for sessions of family therapy, which have provided evidence of a considerable disturbance of family relationships. On this regime, although he physically improved and gained weight, he withdrew from the family to the extent of refusing to talk to his parents, although continuing to live at home.

Despite his continuing problems at home he has begun to show improvement in terms of weight gain (reaching $45 \mathrm{~kg}$ ) and has also demonstrated less disturbance of body image. 
His management has been complicated by multiple admissions with abdominal pain due to recurrent small bowel obstruction. Because of this and an intractable offensive discharge from his mucous fistula he has recently undergone excision of the rectum with ileoanal reanastomosis after creation of an ileal pouch.

\section{CASE 2}

A previously well girl presented at 11.7 years to her local hospital with a three month history of abdominal pain, diarrhoea, and weight loss. Examination showed anal fissures with skin tags and Crohn's colitis was diagnosed on barium enema, sigmoidoscopy, and biopsy. She was treated initially with an exclusion diet, sulphasalazine, and oral prednisolone. She improved symptomatically until her medication was stopped by her parents who had heard of other relatives who had gained weight unacceptably on steroid treatment. Her parents took her to a succession of hypnotherapists, homoeopaths, and other alternative practitioners. During this six month period her general health remained poor and she was unable to return to school, and she received home tuition. At age 12.9 years she frankly relapsed with bloody diarrhoea and hypoproteinaemic oedema. Her parents returned her for review and she was recommenced on prednisolone and sulphasalazine before being referred for further assessment. Colonoscopy and biopsy confirmed patchy Crohn's colitis and barium studies showed no evidence of small bowel disease. Her prednisolone was reduced.

Two months later she was brought to hospital by her grandparents, her parents being unconcerned, because of loss of weight. Her weight had fallen from $40 \mathrm{~kg}$ (above 10th centile) to $32.5 \mathrm{~kg}$ despite good symptom control and no rise of her disease indices. She had become increasingly fussy about her diet and was eating separately from the rest of the family.

She was readmitted for assessment where it became clear that she was avoiding food and secretly exercising. She had a considerable disturbance of body image (figure) and, after formal psychiatric assessment, a diagnosis of anorexia nervosa was made. She admitted that she had become concerned about her weight when she was started on steroids and felt that she had lost control of her own body shape. At

The girl (case 2), who was a talended cartoonist, produced this picture of herself (weight $32 \mathrm{~kg}$ ) and her mother (weight $>50 \mathrm{~kg}$ ).
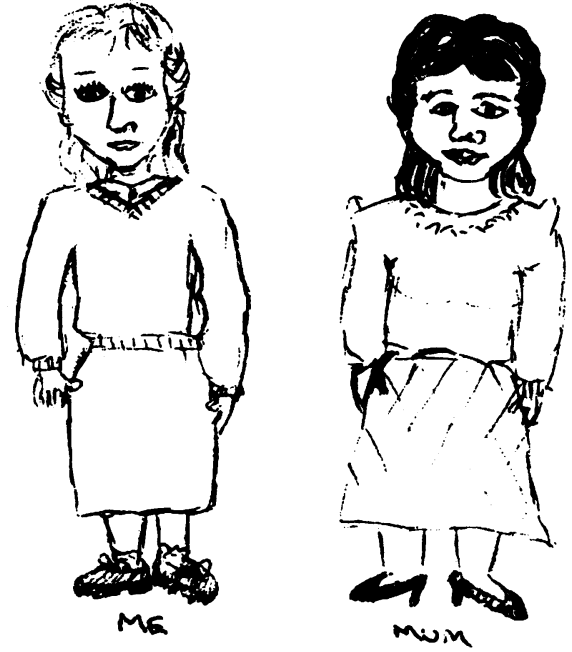

school she had been teased and called 'hamstercheeks'.

Despite an apparent intake of $5.02 \mathrm{MJ} /$ day her weight fell to $29 \mathrm{~kg}$ and her parents eventually agreed to her admission to an adolescent psychiatric inpatient unit. Three units were unable to admit her, two expressing concerns that they would be unable to manage her Crohn's disease (she was currently asymptomatic). Attempts to establish a behavioural regime on the general paediatric ward were partially successful and eventually she was discharged while further attempts were made to find a suitable adolescent unit.

Her disease has subsequently remained quiescent, allowing reduction to low dose alternate day prednisolone. She required admission to her local hospital on one occasion because of food refusal and is currently receiving outpatient treatment at a local family consultation centre. Her weight has improved substantially to $38 \mathrm{~kg}$ and she displays a more normal body image.

\section{Discussion}

Anorexia and weight loss may occur purely as a consequence of inflammatory bowel disease, without any disturbance of body image or other features to suggest a primary disturbance of eating behaviour. ${ }^{2}$ Exacerbation by eating of the abdominal pain due to inflammatory bowel disease may lead to avoidance of a normal diet and consequent weight loss. ${ }^{4}$ The diagnosis of Crohn's disease may be delayed if anorexia and weight loss, without fear of weight gain or body image disturbance, are ascribed to anorexia nervosa. ${ }^{5-7}$ There have been several reports of inflammatory bowel disease presenting as anorexia nervosa, ${ }^{5-8}$ or of anorexia nervosa complicating known inflammatory bowel disease. ${ }^{9}$ All reported cases have been female and in general the diagnosis of anorexia nervosa has been based on the criteria of Dally and Sargant. ${ }^{10}$ These criteria are: (i) that the subject is less than 35 years old; (ii) a refusal to eat; (iii) a more than $10 \%$ weight loss; (iv) an absence of schizophrenia, severe depression, or organic illness; and (v) more than three months amenorrhoea.

These features omit the characteristics psychopathological features of intense fear of weight gain and body image disturbance that are now considered essential for diagnosis. ${ }^{11}$ In addition, there is an erroneous assumption in the older literature that the condition only occurs in young females. Only two of these reported cases would fulfil modern diagnostic criteria for anorexia nervosa. ${ }^{6} 9$

Both our cases show the difficulties in the management of potentially life threatening conditions in what is essentially the wrong setting. The treatment of anorexia nervosa is ideally carried out in a specialist centre with staff experienced in dealing with the unique demands and food avoidance stratagems of these patients. Both our patients were managed in a busy general paediatric ward and were able to circumvent behavioural approaches with relative ease. They would both apparently 
comply with dietary regimes while actually taking a fraction of their documented intake. The boy was frequently reported in the nursing notes to be 'cheerful and eating well'. The girl underwent precise (and much resented) dietary assessment, showing an actual intake of only $1.25 \mathrm{MJ} /$ day instead of the $5.02 \mathrm{MJ}$ she was thought to be receiving. Attempted referral of both patients to appropriate psychiatric units was refused because of concern about the management of organic disease where there was no resident paediatric cover. The boy in particular was able to use his symptoms to forestall an arranged transfer and both would have been able to use their abdominal symptoms to ensure transfer back to the paediatric unit when they became resentful of the management of their eating disorder.

The features and management of each of these two conditions may clearly affect the other. Two cases of Crohn's disease and bulimia nervosa have been described in which corticosteroid treatment affected the role of the eating disorder. ${ }^{12}$ Similarly, in both of our cases, the role of corticosteroid treatment was central. The boy stopped his treatment because of perceived changes in body shape and suffered life threatening consequences. Anorexia nervosa appears to have been precipitated in the girl by the rapid weight gain secondary to high dose steroid treatment. The use of corticosteroids is frequently unavoidable in inflammatory bowel disease, but the risks involved in such treatment at an age when a preoccupation with body shape is common should be clearly understood. The use of alternative management strategies such as elemental diet for small bowel disease should be considered, and patients should be fully counselled about the effects of steroids before they are started. Patients should be followed up regularly and specifically questioned about eating problems. Suspicion of abnormal eating behaviour or unexplained weight loss warrants early formal psychiatric assessment and admission for observation.

We would like to thank Professor JA Walker-Smith for permission to report on his patients and for his kind advice in the preparation of this manuscript.

1 Kelts DG, Grand RJ. Inflammatory bowel disease in children and adolescents. Curr Probl Pediatr 1980;10:1-40.

2 Walker-Smith JA. Crohn's disease and abdominal tuberculosis. Diseases of the small intestine in childhood. 3rd ed. London: Butterworths, 1988:328-60.

3 Bryant-Waugh R, Knibbs J, Fosson A, Kaminski Z, Lask B Long term follow up of patients with early onset anorexia nervosa. Arch Dis Child 1988;63:5-9.

4 Anonymous. Case records of the Massachusetts General Hospital. $N$ Engl f Med 1985;312:1175-83.

5 Jenkins AP, Treasure J, Thompson RP. Crohn's disease presenting as anorexia nervosa. $\mathrm{Br}$ Med $\mathcal{F}$ 1988;296: 699-700

6 Gryboski JD, Katz J, Sangree MH, Herskovic T. Eleven adolescent girls with severe anorexia. Intestinal disease or

anorexia nervosa? Clin Pediatr (Phila) 1968;7:684-90.
7 Hershman MJ, Hershman M. Anorexia nervosa and Crohn's disease. Br $\mathcal{f}$ Clin Pract 1985;39:157, 159.

8 Metcalfe-Gibson C. Anorexia nervosa and Crohn's disease. Br f Surg 1978;65:231-3.

9 Sreenivasan U. Anorexia nervosa associated with energywasting disorders. Can Med Assoc f 1984;130:45-6.

10 Dally P, Sargant W. Treatment and outcome of anorexia nervosa. BrMed F 1966;ii:793-5.

11 American Psychiatric Association. Diagnostic and statistical manual of mental disorders. 3rd ed, revised. Washington, DC: American Psychiatric Association, 1987.

12 Meadows G, Treasure J. Bulimia nervosa and Crohn's disease: two case reports. Acta Psychiatr Scand 1989;79: disease:

\section{Commentary}

Childhood onset anorexia nervosa is often a difficult condition to diagnose and it is frequently overlooked. In one study, the average time from onset to diagnosis was nine months. ${ }^{1}$ The main reasons for the frequent delay in diagnosis are the child's denial and secretiveness. Thus the pathognomonic symptoms such as fear of fat and distorted body image are commonly denied, and other common features such as determined food avoidance, preoccupation with weight and calories, self induced vomiting, exercise and laxative abuse are usually disguised. Anorexia nervosa may all the more easily be overlooked when it complicates inflammatory bowel disease.

In the two patients described by Mallett and Murch, the diagnosis was made on the basis of overt symptoms. In the first case, the boy had a two year history of binge eating, excessive exercising, preoccupation with food intake, weight and body shape, all superimposed upon weight loss. In the second case, the girl had a far shorter history of weight loss, abnormal eating behaviour, and of distorted body image.

How is a diagnosis of anorexia nervosa made in a child with inflammatory bowel disease whose symptoms are far less overt? Firstly, the paediatrician must always be aware of the possibility of a concomitant psychiatric disorder in any child with serious illness. The incidence in such circumstances may be as high as $25 \%$. The regular use of a multiaxial diagnostic scheme such as the International Classification of Diseases ${ }^{2}$ avoids the either/or dichotomy and instead encourages due consideration being given to medical, psychiatric, developmental, intellectual, and social status. Secondly, the paediatrician should have a high index of suspicion when assessing a child with poorly explained weight loss. Certainly the diagnosis should never be excluded on the basis of negative answers to questions about concerns regarding food, weight, and appearance. Thirdly, the diagnosis can almost always be made while the discussion focuses on what would constitute a satisfactory weight, and how much energy should be consumed each day. At this point, children with anorexia nervosa almost always become distressed, argumentative, and negativistic in just the same way as do children whose school phobia has presented with recurrent abdominal pain when told they should now return to school. Finally, if doubt remains, careful observation in hospital will reveal characteristic abnormal eating behaviour and a strong element of secretiveness.

Early diagnosis of anorexia nervosa complicating inflammatory bowel disease is imperative and not only is the condition much harder to treat when treatment is delayed, but as Mallett and Murch have demonstrated it can cause major and even life threatening complications.

1 Fosson A, Knibbs J, Bryant-Waugh R, Lask B. Early onset anorexia nervosa. Arch Dis Child 1987;62:114-8.

2 Rutter M, Shafer S, Sturge C. A guide to a multi-axial classification scheme for psychiatric disorders in childhood and adolescence. London: Institute of Psychiatry, 1975.

B LASK

Department of Psychological Medicine, Hospital for Sick Children, 